\title{
Digital transformation of information related to dental pain management in traditional or complementary and alternative medicine
}

\begin{abstract}
Background: According to the World Health Organization, communication is one of the most important challenges for the integration of Traditional or Complementary and Alternative Medicine (TM/CAM) into healthcare systems.

Objective: To develop harmonized tools and a standardized communication method between traditional and conventional medicine for pain management in order to facilitate communication between health actors in the context of dentistry.

Materials and methods: We took a healer-centered approach and collected 30 information sheets from African healers. Using knowledge engineering techniques we extracted information models, candidate terms and terminologies. Next, we designed a UML-based use case of communication between traditional and conventional medicines. Finally, an integrative communication method was formalized through the identification of processes and communication tools.
\end{abstract}

Results: Our findings showed that patients, dentists and healers communicate using popular terminology that can include specific terms. However, it is possible that they do not fully understand the challenges created by the use of biomedical vocabulary, We were able to develop an integrative communication method structured around a vocabulary shared by traditional medicine and conventional medicine, ontology, a thesaurus, two information models, a context field, the tasks of the health actor, communication interfaces, communication processes and the type of communication.

Conclusion: Communication between traditional medicine and conventional medicine depends on the capacity of healthcare actors and the healthcare system to integrate and use concepts and therapeutic models from the other approach. An evaluation of the ability of a healthcare system and its actors to use the biomedical paradigm from another healthcare system may help to prevent health and socioeconomic risks related to the integration of TM/CAM.

Keywords: world health organization, conventional medicine, complementary and alternative medicine, ontology, biomedical healthcare, communication tools, information models, ontology building
Volume II Issue I - 2018

\begin{abstract}
Martin Sanou, ' Alain Jean, ${ }^{2}$ Michel Marjolet, ${ }^{3}$ Dominique Pécaud, ${ }^{4}$ Yunsan Meas, ${ }^{5}$ Chantal Enguehard, ${ }^{6}$ Leila Moret, ${ }^{7}$ Augustin Emane ${ }^{8}$ 'Doctor of Dentistry, ERT 2004-INSERM U79I, France 2Professor of Dentistry, PU-PH UFR d'Odontologie, France ${ }^{3}$ Professor of Medicine, Laboratoire Pathologie Exotique, France ${ }^{4}$ Sociologist and Philosopher, IHT-Polytech'Nantes, France ${ }^{5}$ Doctor of Medicine, Algologist, Physical Medicine and Rehabilitation Specialist, France

${ }^{6}$ Natural Language Processing Specialist, UMR CNRS 624I, France

'Doctor of Medicine, Biostatistics and Pharmacoepidemiology Specialist, France

${ }^{8}$ Professor of Social Rights, UFR Droits et sciences politiques, France
\end{abstract}

Correspondence: Martin Sanou, Doctor of Dentistry, ERT 2004-INSERM U79I, Nantes, France, Email martinsanou@gmail.com

Received: February 06, 2018 | Published: February 16, 2018
Abbreviations: WHO, world health organization; CAM, complementary and alternative medicine; $\mathrm{CM}$, conventional medicine; $\mathrm{TM}$, traditional medicine

\section{Introduction}

In 2002 the World Health Organization (WHO) defined its first global strategy for Traditional and Complementary and Alternative medicine (TM/CAM).$^{1-4}$ The scientific evidence provided by various integrative strategies ${ }^{5-7}$ show that the majority of healthcare systems in Africa and elsewhere are seeking to develop TM/CAM. The WHO's systemic approach aims to integrate traditional healers into healthcare systems and promote collaboration between the physician and the traditional healer in order to improve the health of the population. ${ }^{1-5}$

Communication is one of the most important challenges for TM/ CAM integration into healthcare systems. Experts agree that in order to maximize the potential of TM/CAM in health care, several issues must be addressed: national policies and regulatory frameworks, safety-efficiency and quality, access to both therapies and products, and the lack of information available to the public on its use. Another specific challenge is the lack of communication between traditional healers, physicians and consumers. ${ }^{1}$

The pan-European Cambrella project highlighted the key role of good information, harmonization of terminology and controlled vocabulary in the use of TM/CAM. ${ }^{8,9}$ Countries vary widely in the extent to which TM/CAM has been integrated. ${ }^{5,8,9}$ Despite the lack of research-based knowledge into the attitudes and needs of people regarding TM/CAM in many European countries, some trends can be noted. TM/CAM is widely accepted and many people would like it to be made more available. They would like impartial, reliable and trustworthy information that supports informed decision-making, and more support and involvement in biomedical healthcare choices. ${ }^{8,9}$

Both communication and information about TM/CAM are fundamental in the evaluation of TM/CAM risk. There is extraordinary diversity in the way that TM/CAM practices are regulated around the world. ${ }^{1,9}$ Consequently, the lack of high-quality information and communication may lead to medical risk, socio-economic problems 
and a failure to integrate TM/CAM into healthcare systems. ${ }^{9,10}$ Online biomedical data on treatments for various diseases and alternative therapies ${ }^{11}$ is available at various levels. Examples include the French Académie Nationale de Médecine (National Medical Academy), ${ }^{12}$ healthcare organizations such as the French Mission Interministérielle de Vigilance et de Luttes contre les Dérives Sectaires (the Interministerial Taskforce for Monitoring and Combatting Sectarian Aberrations) ${ }^{13}$ national and international agencies for the safety of medicines and health products (e.g. the European Medicines Agency) ${ }^{14,15}$ or the American Food and Drug Administration. ${ }^{16}$

The harmonization and standardization of communication with $\mathrm{TM} / \mathrm{CAM}$ is a new challenge for all healthcare systems and actors, and integrative policies have not yet fully addressed the problem. ${ }^{2,5}$ Structured information about TM/CAM could facilitate communication between consumers, traditional healers and physicians. ${ }^{2,9}$ Furthermore, the goalposts regarding the use of TM/ CAM are constantly moving. ${ }^{2,5}$ Its growing popularity has resulted in the appearance of new TM/CAM products and therapies. $2,5,9,17,18$ This increased competition in the under-regulated TM/CAM market is accompanied by new systems of marketing, communication and advertising. ${ }^{2,5,9,13,14}$ Finally the new concept of "associated" medicine demonstrates that all European healthcare systems can learn from the African system. In this context, standardized terminology and controlled vocabulary are fundamental to the exchange of information between the two systems. ${ }^{10}$

We selected dental pain as the subject for this study ${ }^{10}$ as patients have similar motives for consulting a practitioner whether in the conventional, integrative or TM/CAM system. Pain is one of the oldest medical problems and a universal physical affliction for humankind. ${ }^{19}$ It is a concept that is common to both TM/CAM and conventional medicine and is one of the most frequent reasons for consulting either a traditional healer or a biomedical practitioner. Some TM/CAM therapies are well-known for their effectiveness in managing pain. For example, acupuncture has been used to treat pain (and many other health conditions) for more than 3,000years. ${ }^{20}$ In Africa, traditional healers play an important role in the delivery of primary health care, particularly in remote communities ${ }^{21,22}$ and offer various approaches to pain management. Their traditional methods range from plant remedies to tooth extractions. The question we investigate in this study is whether it is possible to develop homogeneous tools for clear communication between African healers, patients, and actors in conventional medicine for the management of dental pain.

The aim is to develop standardized tools and a harmonized communication method between African traditional medicine and conventional medicine for the management of dental pain. It seeks to facilitate communication between actors in the healthcare sector in the context of the World Health Organization's strategy to integrate TM/ CAM into mainstream medicine.

\section{Materials and methods}

\section{The communication framework}

The starting point for our study was an ideal communication use case. In this first example, we examined two healthcare systems, two models of medicine, three actors (the healer, the patient, and the practitioner) and a biomedical information system (Figure 1). The prototype was based on the challenges identified by the WHO related to strategies for the integration of traditional medicine into healthcare systems. ${ }^{2,5}$ We also looked at bibliographic data on clinical trials, and programs to promote traditional medicine in the treatment of various pathologies. ${ }^{5,6,8}$ The goal was to identify a comprehensive set of variables and constraints related to communication; to detect communication mechanisms; and to find links between traditional medicine and conventional medicine that went beyond a strict comparison.

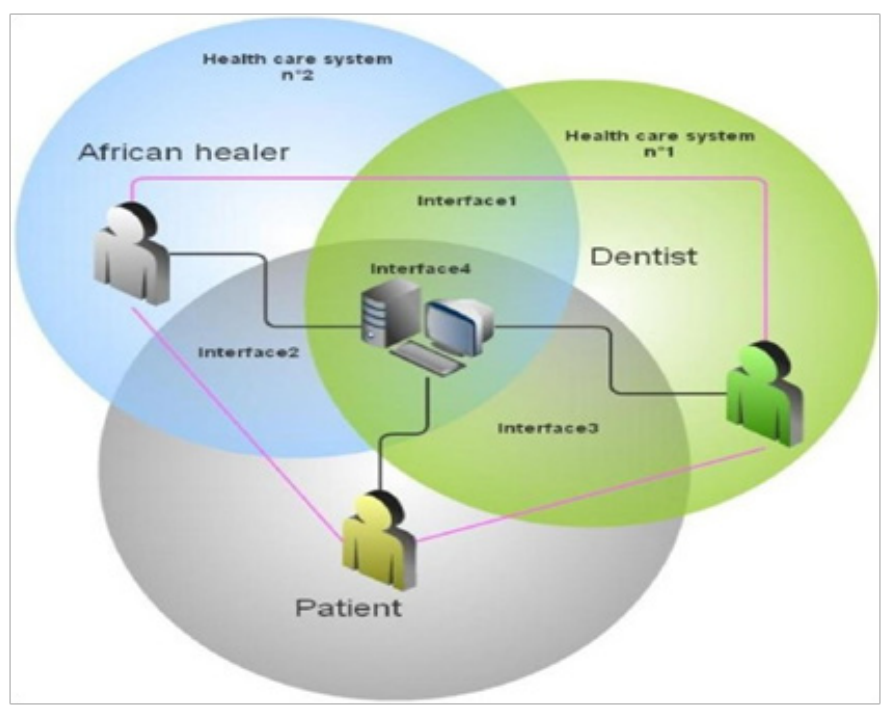

Figure I A communication use case involving TM/CAM and the CM healthcare system.

\section{Collection and analysis of African healer's terminology}

From 2002 to 2011, we collected 30 commercial information sheets from African traditional healers in various countries (Benin, Burkina Faso, Gabon, Gambia, and Mali). These paper documents provided a range of information about the healers and their services such as: the therapies available, the illness or problems treated, and the medical products used. From these information sheets, we extracted frequently-used terms using natural language processing procedures. A corpus of 10,800 words was established, unidentifiable words were checked for spelling mistakes, and the text was pre-processed using a corpus processing system (Unitex 2.1). Terms were extracted manually according to token frequency, the identification of collocations, the use of patterns for locating information, and statements about the definition of pain. ${ }^{23}$ We then identified the principal pathologies through a correlation of term frequencies and the concepts of healers. We also categorized terms indexed in conventional biomedical terminologies to identify semantic differences between socioeconomic or cultural problems, and disease or illness. ${ }^{10}$

\section{Communication tools}

Development of a common terminology: The terms that were identified were recorded in a joint TM/CAM and conventional medicine terminology bank. These records were organized according to: class, domain, division or field of the term, definition, related terms and synonyms, equivalents in other languages, the source of word, history, and additional purposes. ${ }^{24-26}$

\section{Information models}

The purpose of extracting information compiled from information sheets was to enable it to be shared between computer systems and 
health professionals. Therefore we modeled information entities in an information domain model. The first version of this model was named MIDOMA-alpha.1. Then, on the basis of a definition of use case communication scenarios, we designed the second information model (MIDOMA-beta.1). From this we were able to extract items that demonstrated the relationship between traditional medicine and conventional medicine. The class diagram was constructed using UML formalisms. ${ }^{27,28}$

Ontology building: At this point we reused approaches taken in the development of other ontology projects (MENELAS ${ }^{29}$ and ONTOORPHA $^{30}$ ). We determined the levels of the ontology (top- or upper-level, core, and domain), its classes and class hierarchy. ${ }^{25,31} \mathrm{~A}$ knowledge representation language (the Ontology Web Language, OWL) was selected to operationalize the ontology. Protégé, a free open-source ontology editor and knowledge-based framework was used to analyze it. ${ }^{32}$

Thesaurus building: Our approach to the construction of a controlled vocabulary synthesized work carried out in other studies. ${ }^{33-37}$ First we selected descriptors (terms). Then we identified most frequent ontological relationships of each term (associative, hierarchical). Experts in the field of alternative medicine provided an opinion on the definition and meaning of certain concepts. Although these experts had an intellectual and academic profile they had also acquired traditional knowledge. Finally, a thesaurus was constructed semiautomatically using software (Thesaurus Builder professional version 6.5.5.1) which is compliant with ISO 5964..$^{38}$

Identification of communication processes: In order to standardize communication between TM/CAM and conventional medicine practitioners we defined communication processes. The main constraint was that these processes should be accessible to all actors including the patient, the traditional healer and the clinician. Therefore, we undertook a preliminary survey of the perception of traditional concepts in the conventional medicine setting. The survey was administered to 78 students, teachers and patients of a dental care center in Nantes (western France). The survey looked at the ability of actors to use concepts from a different health system taking into account socio-cultural, linguistic, semantic and terminological factors.

Communication typology: A typology of communication between $\mathrm{TM} / \mathrm{CAM}$ and conventional medicine (CM) had been identified on the basis of published articles and information available on the Internet. ${ }^{8,9,11}$ This showed a relationship between a lack of communication and the challenges of integrating traditional medicine. Although any healthcare system can integrate traditional medicine, the means of communication are highly variable. ${ }^{2-6}$ In addition, communication in a healthcare system depends on an exchange of concepts and therapeutic or explanatory models. This exchange is guided by the use of terminology and the context for the use of traditional concepts. Such forms of communication are not specific to dental pain and can also be applied to other pathologies.

\section{Results}

\section{Harmonized communication tools Information models}

The MIDoMA-alpha.1 information model was derived from the traditional healers' information sheets, and the initial constraints for the development of a standardized communication method between conventional and traditional medicines were established. MIDoMAalpha. 1 describes the basic information exchanged in a workflow that involves the patient, the healer and the CM doctor. UML classes 27,28 (at this stage without attributes and association rules) described medical, social, economic and geographic information. This information included: the healer's skills, patient needs and the potential healing power of the treatment (Figure 2).



Figure 2 The MIDoMA-alpha.I information model developed from documentation provided by traditional healers. 
The MIDoMA-beta. 1 information model was based on biomedical concepts. It outlined non-exhaustive lists of communication variables between TM/CAM and CM. It provided a better view of the concepts, explanatory models, paradigms and reasoning to be integrated by healthcare actors before the implementation of a strategy for incorporating TM/CAM into the healthcare system (Figure 3).

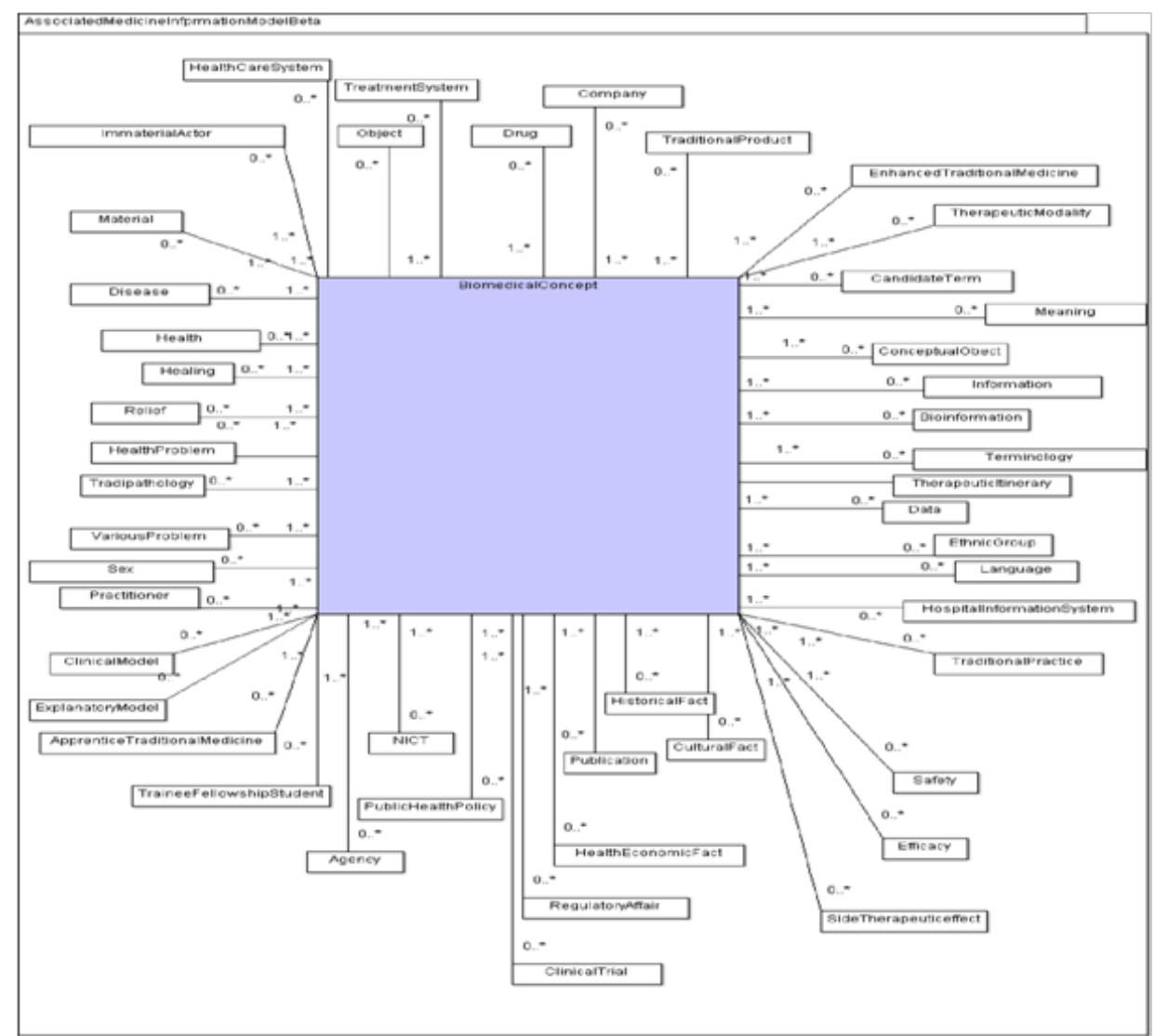

Figure 3 The MIDoMA-beta.I information model based on biomedical concepts.

\section{Records of terms}

The record containing the description of a term (Table 1) made it possible, for each candidate term, to assess the uniqueness of a concept. For example, the term "toothache" or "dental pain" (a rough translation of maux de dent in French) is defined by a list consisting of fields, labels, and their contents. The number of concepts, classes, divisions and sections was also recorded. It should be noted that the terms mal and maux are meta-concepts that are frequently used by French-speaking African healers. Their meaning may include illness, disease, pain, suffering, evil, etc.

Table I Dental pain (maux de dent) record with selected fields

\begin{tabular}{ll}
\hline Field & Contents \\
\hline Class & Associated Medicine \\
Division & Knowledge \\
Section: E & Pain or pains \\
Section: N & Tooth anatomy \\
Language & French \\
& Definition from a healer's information sheet: \\
& Tooth decay: Toothache due to transient or chronic pain \\
& Official publication: National Health Service, United Kingdom, Patient \\
and general public information sheet I' & Medical Subject Headings: Odontology \\
Definition & Toothache: Dental pain is pain in the jaw and face. It is usually due to a dental problem. \\
& Dental pain can be caused by nerve irritation in the root, infection, and decay or tooth loss. \\
& Toothache \\
Synonyms and pseudo- synonyms & Buccodental pain Odontology \\
\hline
\end{tabular}


Table Continued....

\begin{tabular}{|c|c|}
\hline Field & Contents \\
\hline $\begin{array}{l}\text { Medical interpretation or } \\
\text { therapeutic decision }\end{array}$ & Potential positive diagnosis: pulpitis, periodontitis, maxillary cellulitis \\
\hline Conceptual indicators & Pain \\
\hline Linguistic indicators & French \\
\hline Terminology indicators & Uncoded: SNOMED, CIM I0, MedDRA French code: CisMEF \\
\hline Regulatory indicators & Not authorized for market use \\
\hline Health actor indicators & Healer Clinician \\
\hline Other usage & Term identified during a 201 I survey with 40 dentists in Nantes, France \\
\hline Gender & Male \\
\hline Symbols-pictograms & - \\
\hline Normalization & - \\
\hline
\end{tabular}

'http://www.nhs.uk/translationfrench/Documents/Toothache_French_FINAL.pdf

In the specific context of dental pain management, the "tooth" was described as an organ. Candidate terms describing the tissues that made up the dental organ did not appear in the corpus; these include terms such as dentin, cementum, desmodontium, pulp, periodontal ligament, alveolar bone and cellulosic adipose tissue. Similarly, alternative terms, phrases and collocations were completely missing such as: "acute", "toothache", "pulpitis", "acute pulpitis", "periodontitis" and "maxillary cellulite". On the other hand, concepts found in the corpus, such as "dental diarrhea" (diarrhée dentaire) are unknown in conventional medicine. It seems that traditional healers struggle to understand that certain types of dental pain are related to inflammation. Infectious conditions are described in terms of "infection" and "abscess" and are not directly related to the odontalgia.

\section{Ontology}

Using the candidate terms derived from our terminology research, we built an ontology that included both traditional and conventional terms. This first version consisted of 67 ontological classes (the toplevel ontology) and was six levels deep. For example, the hierarchy used to define the concept of toothache (maux de dent) was structured as follows:

i. Top-level ontology (general concepts, thinking and philosophy): the concept of pain (la notion du mal);

ii. Core ontology (structural concepts): pains, pains (mal, maux); and

iii. Domain ontology (domain concepts): dental pain (les maux de dent, le mal de dent). The pain (maux) concept formed part of the super-class called dental pain (maux de dent) (Figure 4).

\section{Thesaurus}

The Thesaurus of Associated Medicine (MAThes) consists of ten semantic fields:

a. Health actors: this field includes all stakeholders in the healthcare system. It targets those receiving treatment and caregivers (patients, healers, and healthcare professionals);

b. Traditional and conventional knowledge: the data, information and practices found in traditional and/or conventional medicine;

c. The context: defined by the medical environment and the familial, social, economic, anthropological, linguistic and geostrategic context of a clinical case or pathological condition;

d. The effects: both therapeutic effects and any socio-economic and public health impacts. Effects can be behavioral, cognitive, and psychological in the context of an individual patient;

e. Clinical and explanatory models: the traditional or conventional patterns that have been identified for describing and managing diseases;

f. Medical and biomedical models: the type of medicine, skills, practices and disciplines with which the patient is managed (e.g. integrative medicine, chemotherapy or acupuncture);

g. Point of view: the transversal and interdisciplinary characteristics of problems related to TM/CAM integration from which emerge ideas about health problems. These could be anthropological, historical, social, economic or medical;

h. Objects and products: these describe traditional or conventional 
drugs, traditional medicines and the various products referred to in pharmacopeia (plants, herbs, bark, solutions, etc.)

i. TM/CAM integration strategies: these include national and international policies on TM/CAM integration. At a local level, they concern programs created by traditional healers associations and non-governmental organizations for the development of TM/CAM;

j. Healthcare systems: these define how healers and practitioners are organized in a healthcare system at the level of the individual and the group, and national healthcare infrastructure (Figure 5).

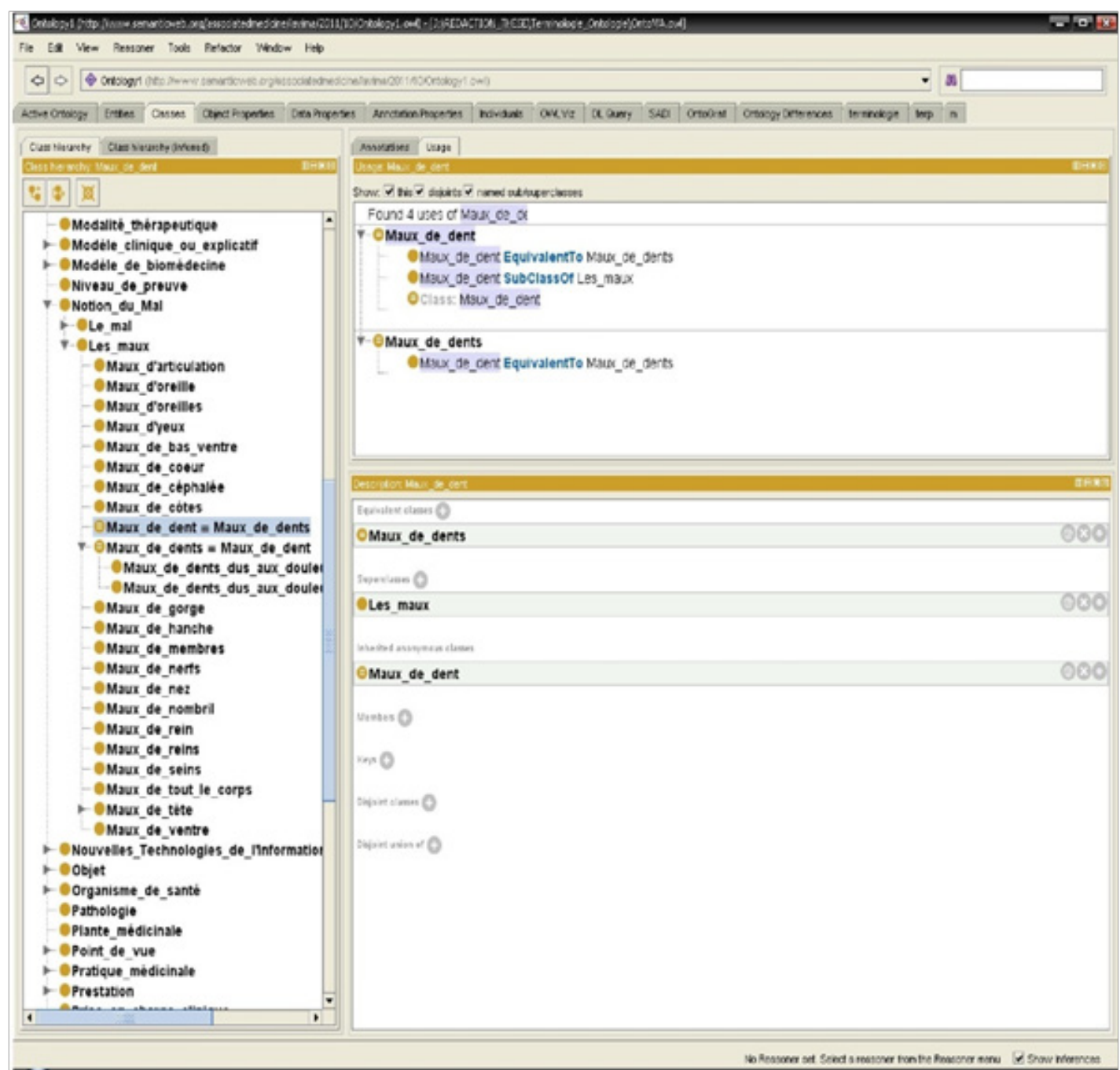

Figure 4 The ontology created using Protégé

\section{Standardized communication between TM/CAM and CM theoretical framework: associated medicine}

We defined and described a new concept of public health known as associated medicine. It looks at the capacity of a healthcare system and its actors to associate or be associated with a model of medicine. It explains how a healthcare system " $A$ " can learn from healthcare system "B". Put another way, it describes how one healthcare system can integrate a therapeutic paradigm from another healthcare system. The principal aim was to measure the capacity of a healthcare system to integrate the following: biomedical concepts, biomedical facts, therapeutic or biomedical models, biotechnology from a single type of biomedicine (associated medicine) or from many distinct models of medicine (associated medicines). The second aim was to examine the capacity of the system and its actors to create socio-economic, public health and therapeutic benefits. Other objectives were: to evaluate the consequences for human health and the organization of healthcare systems; to describe interactions between healthcare systems; and to analyze changing dynamics in public health crises

On a collaborative note, the framework offers a way to manage the flow of information between healthcare actors. It favors the integration of traditional medicine into conventional medicine through communication, and can optimize strategies for the integration of traditional medicine into another healthcare system. At a clinical level, it can help in the integration of modalities and conditions of care, and to clarify medical risk management. In pharmacology, it can demonstrate trends in the evolution of concepts associated with a pharmaceutical product during its biotechnological and therapeutic lifespan. At an individual level, it focuses on the behavioral, cognitive and psychological changes linked to a biomedical concept through a conceptual approach to medical facts. It takes as its object concepts that represent sickness, health, medicine, patients, CM practitioners, TM practitioners and the healthcare system. ${ }^{10}$ 


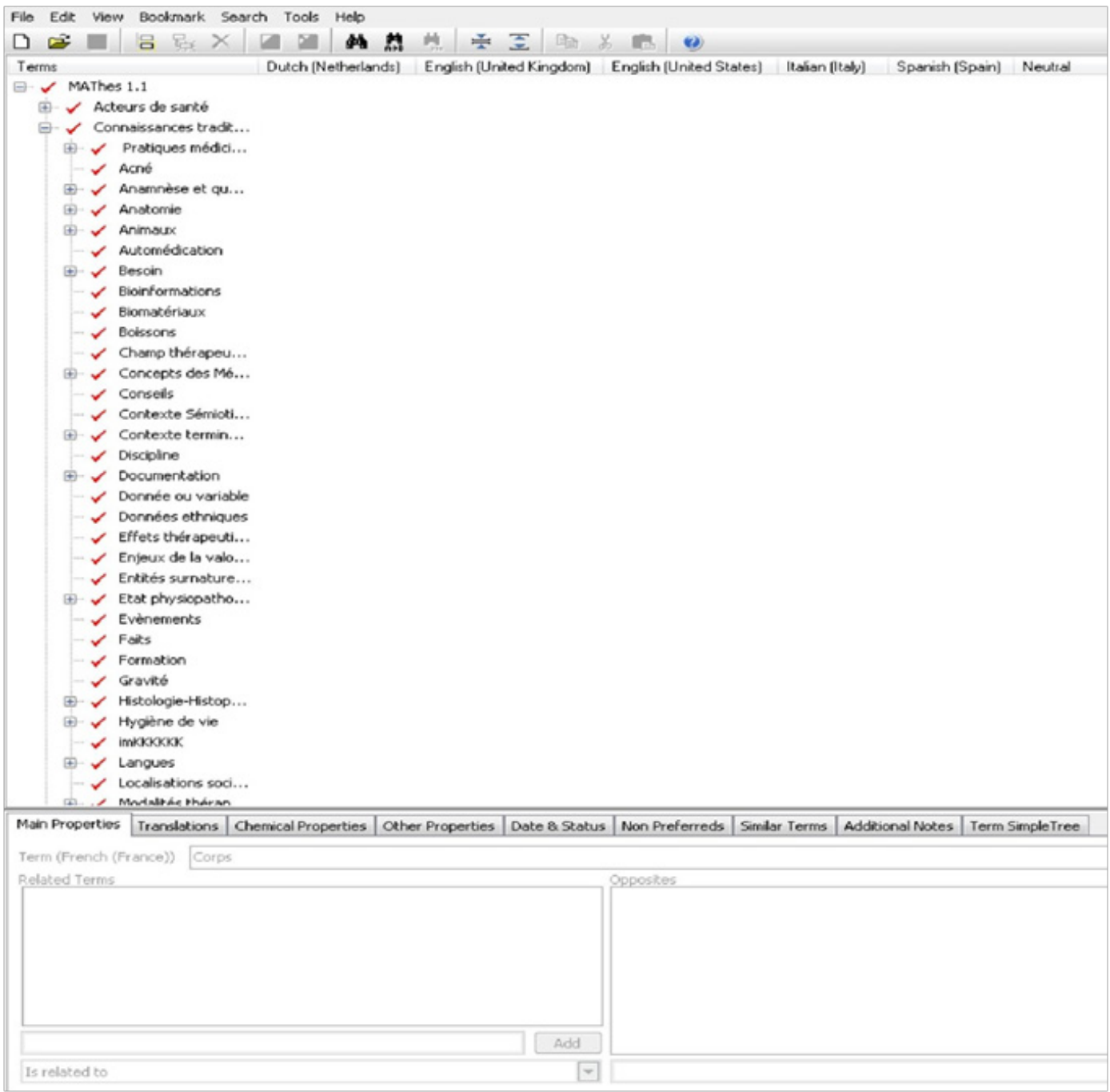

Figure 5 The thesaurus created using Thesaurus Builder.

\section{Communication processes}

The conceptual framework was based on the results of pre-surveys and investigations. It was organized into four main areas: resources and communication, healthcare actors, treatment of dental pain, medical risk management and contextual concepts related to disease. The creation of communication processes was based on a selection of all predicates and verb phrases that characterized the speech and therapeutic procedures of a healthcare actor. In African traditional medicine the oral tradition is very important in the transmission of knowledge and it also features in conventional clinical communication. We named it the "standard exchange format" or the "oral format" because the spoken word is easily understood by all healthcare actors.

The medical phrases and behaviors that were identified in this way were combined into pairs of opposites: write - do not write; know do not know; care - do not care; heal - does not heal; relieve - does not relieve; help - does not help; believe - do not believe; use - do not use; treat - does not treat, understand - do not understand; know - do not know; see - do not see; read - do not read (Figure 6).

\section{Communication activities}

Communication activities consisted of tasks related to communication between healthcare actors:

i. Determining the communication interface: man-machine, manman, patient-healer, healer-practitioner, practitioner-patient, etc.;

ii. Identifying the context: management of dental pain;

iii. Selecting the communication process;

iv. Identifying communication resources (terminology, ontology, thesaurus) in order to deduce communication options;

v. Selecting the type of communication (possible, impossible, juxtaposed, regulated, consensual, etc.).

vi. Identifying shared information: information on $\mathrm{CM}$ and/or mystical-religious therapeutic practices.

Usage rules and codes for communication methods varied. There was no fixed entry point; it dynamically depended on communication needs and the appearance of terms describing diseases or other terms that were unrecognized in the therapeutic procedures of health authorities. Such terms did not belong to the official nomenclature. For example the concept of "help" was considered as "known" but not "written" in the speech of holistic healers (Figure 6). 


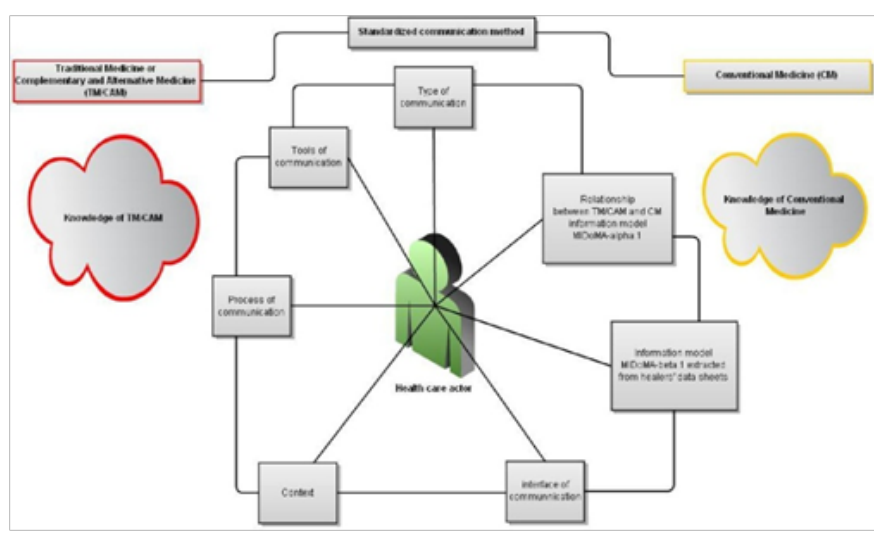

Figure 6 The standardized communication scheme.

\section{Discussion}

We could have decided to orient our methodology towards ethno-linguistics and ethnopharmacology. ${ }^{39-43}$ However, we instead chose to focus on traditional knowledge engineering techniques and technological solutions. The main reasons for this are the explosion of information that is available on the Internet and social networks about alternative medicine and its integration into CM. ${ }^{11,44}$ In addition, anthropological and ethnological methods require considerable time in order to gain the trust of traditional healers. These factors led to our method which focuses on the technical and commercial aspects of the work of traditional healers.

Our work may have sociological limitations. The question arises of whether it is even useful to compare data from African healers to that of European witchdoctors (marabouts) or French dentists? Logically these three sets of actors correspond to three different approaches to health and wellbeing. The complexity of the issue means that it is important to be prudent in any sociological generalization of our results. The only point that these healthcare actors have in common is the fact that their skills (secular or not) are recognized in their social environment. Nevertheless, our results showed that the concepts of pain and dental pain ( $m a l$ and maux de dent) are very popular themes in both Africa and Europe. Word-of-mouth and the Internet are effective vectors for the propagation of such concepts. ${ }^{11-15}$

Capturing the knowledge and methods of traditional healers formed the cornerstone for our research. Nevertheless, the knowledge of healers can be said to be mystical, supernatural and difficult to formalize..$^{10}$ A legitimate question is how we were able to collect such data. We initially focused on written words and practices. However, we also included healers' spoken language, which made it possible to integrate the oral characteristics of African traditional medicine. Consequently, the size of the corpus was quite small compared to others that consist of millions of words ${ }^{45,46}$ Full details of how healers' information sheets were encoded are beyond the scope of this paper. ${ }^{47}$

The standardized terminology tools we developed may appear complex when applied to traditional healers, especially as some healers do not speak French or any other occidental language (although sometimes they speak "African French", a combination of French and the local language ${ }^{48}$ ). Our communication method is oriented towards healthcare actors from both conventional and traditional medicines. The terminology, ontology and thesaurus we constructed attempted to respect the multilingual character of TM/CAM. For some terms it was necessary to find a compromise. The translation of a concept such as dental pain (maux de dents) was just as difficult in African languages as it can be in French or English. It can be used to refer to pain, pathology or the "bad spirit" that is the case of a disease. ${ }^{10}$ For all of these reasons, there is a need for tools and indicators that facilitate the use of terminology resources. Simplified terminology tools need to be developed and implemented for novice users of the communication method.

Interviews with dentists about their use of clinical terminology showed that they tend to avoid certain words (such as healers ${ }^{10}$ ). We also noted mismatches in the semantics of particular groups of words. For example, some dental practitioners avoid the words "devitalization" and "dead" to describe teeth because patients are sensitive to these terms. This led to issues related to the meaning of the words we collected. For example, is it better to say "devitalized tooth" or "devitalized pulp"; what about "painful decay"? Can we devitalize a "dead tooth"? Is it better to "remove the pulp" or "remove the nerve"? We did not analyze other esoteric concepts or dental methods, such as magic, dental decoding, psychodental analysis or dental energetics.

This study showed that it is possible to provide evidence of the efficacy and safety of a medicinal product ${ }^{1}$ simply with words. The strength of our method is that it can demonstrate socio-economic and medical risks on the basis of a knowledge representation of the elements of integrated communication. This may help to promote the implementation of pharmacovigilance policies and precautions for the use of traditional products. ${ }^{1,2,8}$

Although we have not clinically tested our communication method, we have assessed the socio-economic and health risks found in healer's information sheets. In this part of the study we checked the consistency of the information provided, taking into account the traditional character of TM/CAM integration (the oral tradition, the local language and ethnicity). We addressed this issue in terms of the concept of quality which is itself a concept used in conventional medicine. ${ }^{1,49}$ The criteria and scoring of medical and biomedical information was based on the Health on the Net (HON) Code of Conduct, ${ }^{50,51}$ Netscoring, ${ }^{52}$ the Dublin Core ${ }^{53}$ and Cimino ${ }^{54}$ However, several difficulties in the use of these criteria were identified as, in practice, they tend to be oriented towards information found on the Internet and in hospital information systems. We therefore decided to assess the paper-based documents used by traditional healers (Table 2).

Table 2 evaluating healers' data sheets by criteria to assess the quality of health internet information

\begin{tabular}{lll}
\hline Referentials standards & Evaluation criteria & compliance of terminological resources of healers \\
\hline HONcode & Authoritative & yes \\
& Complementarily & yes \\
& Privacy & non controlled \\
& Attribution & sometimes unknown data \\
\hline
\end{tabular}


Table Continued...

\begin{tabular}{|c|c|c|}
\hline Referentials standards & Evaluation criteria & compliance of terminological resources of healers \\
\hline \multirow{39}{*}{ James Cimino } & & sources \\
\hline & Justifiability & rational and irrational \\
\hline & Financial disclosure & relative \\
\hline & Transparency & non controlled \\
\hline & Advertising policy & no warranty \\
\hline & Completeness & released only for all health \\
\hline & & actors \\
\hline & Concept orientation & multiaxial \\
\hline & Concept permanence & evolution of concepts \\
\hline & Polyhierarchy & yes \\
\hline & Formal definitions & relate only to indexed candidate terms \\
\hline & Reject "not elsewhere classified" & relative.the « african french » is accepted \\
\hline & Multiple granularities & yes \\
\hline & Multiple Consistent Views & yes \\
\hline & Beyond medical concepts: & yes \\
\hline & representing context & \\
\hline & Recognize Redundancy & yes \\
\hline & Title & name of healer, list of products, plants or diseases \\
\hline & Creator & individuel ou associatif ou \\
\hline & & institutionnel \\
\hline & Subjects or keywords & yes \\
\hline & Description & yes \\
\hline & Publisher & non-systematic \\
\hline & Contributor & social environment is \\
\hline & & sometimes literate \\
\hline & Date & uncommon \\
\hline & Type of resource & paper documents, banners, \\
\hline & & flyers, technical data sheets, \\
\hline & & advertising panels, visit cards \\
\hline & Identifier of resource & non-systematic \\
\hline & Source & not always identifiable \\
\hline & Language & multilingualism \\
\hline & Relation & partial indexation of some \\
\hline & & candidate terms \\
\hline & Coverage & village or regional, national and international coverage \\
\hline & Intellectual Property Rights, & hard to define \\
\hline & Copyright, various rights & sometimes organized by health authorities or associations \\
\hline & Credibility & relative \\
\hline & Content & inconstant \\
\hline
\end{tabular}


Table Continued....

\begin{tabular}{lll}
\hline Referentials standards & Evaluation criteria & compliance of terminological resources of healers \\
\hline Hyperlinks & usually inexistent \\
Design & simple & multiplicity of traditional \\
& symbols \\
& relative \\
Quantitative aspects & dominant oral tradition bit of paper documents \\
Ethics & no warranty \\
Accessibility & popular knowledge \\
& numerical contents of african healer data sheets are less available on the internet
\end{tabular}

Finally, there are many challenges to be overcome if practitioners of the various CAM/TM therapies are to understand each other's concepts. However, we did not compare the terminology and concepts of different forms of TM/CAM. Instead, we investigated commonalities, differences and invariants between East and West, and South and North in the use of terms derived from TM/CAM. Concepts and approaches from acupuncture, homeopathy ${ }^{55}$ and African traditional medicine do not correspond to one communication mechanism or a single spoken language, although similar issues apply to the labelling, marketing and communication of TM/CAM products. ${ }^{56}$ In the context of complementary and alternative medicine the goalposts are constantly moving. Its growing popularity has led to the emergence of new specialties and growing medical, social, economic and commercial competition. ${ }^{2,5,8}$ In this context, our study may facilitate further work into new systems for the marketing, communication and publicity surrounding TM/CAM in the next decade.

\section{Conclusion}

The construction of standardized tools to enhance communication in integrative pain management led to the emergence of metaconcepts which highlight the huge communication challenges for the integration of TM/CAM. An example is the concept of "pain", which is frequently used by traditional healers to describe both general pathology and specific disorders. It can also be used to refer to social, cultural, economic and spiritual conditions and symbolizes all aspects of the biopsychosocial pain model. Similarly, the study demonstrated linguistic and semantic differences between the French definition of the concept of mal and the term "pain" in English. This finding could be a predictor of the cultural and anthropological characteristics of integrative pain management in European countries. As a consequence, it may be the case that some African medical practices, skills and therapies are too complex to integrate into conventional healthcare systems worker and businessman. Communication activities consist of tasks related to communication between healthcare actors. They include: determining the communication interface (man-machine, man-man, patient-healer, healer-practitioner, practitioner-patient, etc.); identifying the context (the management of dental pain); selecting the communication process; identifying communication resources and methods (terminology, ontology, thesaurus); selecting the type of communication (possible, impossible, juxtaposed, regulated, consensual, etc.); and identifying shared information (related to $\mathrm{CM}$ and/or mystical-religious therapeutic practices).

The digital transformation of information related to traditional medicine or complementary and alternative medicine is a challenge for scientists and future generations. Indeed, the information of traditional medicine sometimes has supernatural values. We have suggested in these work lines of reasoning and systems of thought by proposing interdisciplinary and transdisciplinary approaches. In the future, every aspect of this study could be the subject of intradisciplinary scientific research.

\section{Acknowledgements}

None.

\section{Conflict of interest}

The author declares no conflict of interest.

\section{References}

1. World Health Organization (WHO). General guidelines for methodologies on research and evaluation of traditional medicine. WHO/EDM/TRM/2000, 1, Geneva: WHO; 2000. p. 1-17.

2. World Health Organization (WHO). WHO traditional medicine strategy 2002-2005. WHO/EDM/TRM/2002.1, Geneva: WHO; 2002. p. 1-74.

3. World health organization congress. Déclaration de Beijing. 08-2366FTRM, Geneva: World Health Organization; 2008.

4. World Health Organization (WHO). Rapport de situation sur la décennie de la médecine traditionnelle dans la région africaine. AFR/RC61/PR/2, Yamoussoukro. Geneva: World Health Organization; 2000. p. 1-6.

5. Johnson C. Health care transitions:a review of integrated, integrative, and integration concepts. J Manipulative Physiol Ther. 2009;32(9):703-713.

6. UNAIDS. Collaborating with traditional healers for HIV prevention and care in sub-Saharan Africa: suggestions for programme managers and field workers. UNAIDS best practice collection. Geneva: UNAIDS; 2004. $54 \mathrm{p}$.

7. World Health Organization (WHO). Traditional medicine. Factsheet $\mathrm{N}^{\circ} 134$, Geneva: WHO media centre; 2008.

8. http://www.cambrella.eu/home.php.

9. Walach $\mathrm{H}$, Weidenhammer W. Insights into the Current Situation of CAM in Europe: Major Findings of the EU Project CAMbrella. KARGER et al editors. Forschende Komplementärmedizin/Research in Complementary Medicine. 2012;19(2).

10. Sanou M, Jean A, Marjolet M, et al. Conventional medical attitudes to using a traditional medicine vodou-based model of pain management:survey of French dentists and the proposal of a pain model to facilitate integration. J Chiropractic Hum. 2012;19:24-35. 
11. Keshet Y. Network gatekeeping:Complementary medicine information on the websites of medical institutions. Health (London). 2012;16(2):15168.

12. Bontoux D, Couturier D, Menkès CJ. Les thérapies complémentaires - leur place parmi les ressources de soins. Rapport, Paris: Académie Nationale de Médecine; 2013.

13. http://www.miviludes.gouv.fr/IMG/pdf/ra2010miseenligne.pdf.

14. ANSM-Afssaps. Rapport annuel:Activité de contrôle de la publicité du médicament et des autres produitsd santé 2009; 2010.

15. Committee on Herbal Medicinal Products, European Medicines Agency. Procedure on the publication of HMPC public statements when Community herbal monographs on herbal substances, preparations and/ or combinations thereof are not established. EMA/HMPC/84530/2010. London; 2010.

16. Roller ST, Pippins RR, Ngai JW. FDA's expanding postmarket authority to monitor and publicize food and consumer health product risks:the need for procedural safeguards to reduce "transparency" policy harms in the post-9/11 regulatory environment. Food Drug Law J. 2009;64(3):577598.

17. Nahin RL, Barnes PM, Stussman BJ, et al. Costs of Complementary and Alternative Medicine (CAM) and Frequency of Visits to CAM Practitioners:United States, 2007. Natl Health Stat Report. 2009;18:114.

18. Lazarus A, Delahaye G. Médecines complémentaires et alternatives:une concurrence à l'assaut de la médecine de preuves? Les Tribunes de la santé. 2007;2(15):79-94.

19. Meldrum ML. A capsule history of pain management. JAMA. 2003;290(18):2470-2475.

20. Dorsher PT. Acupuncture for chronic pain. Tech Reg Anesth Pain Manag. 2013;15(2):55-63.

21. Agbor AM, Naidoo S. Knowledge and practice of traditional healers in oral health in the Bui Division, Cameroon. J Ethnobiol Ethnomed. 2011;7:6.

22. Agbor Ashu M, Naidoo Sudeshni, Mbia Awono M. The role of traditional healers in tooth extractions in Lekie Division,Cameroon. $J$ Ethnobiol Ethnomed. 2011;7:15.

23. http://www-igm.univ-mlv.fr/ unitex/.

24. Pavel S and Diane Nolet D. Handbook of terminology. Terminology and standardization. Translation Bureau. Québec; 2001. p. 1-83.

25. Roche C. Terminologie et ontologie. Langages; 2005. p. 48-62.

26. http://www.vidal.fr/vumef/fichiers/PublicationsDiffusees/ ZweigenbaumISIS99.pdf.

27. http://www.omg.org/gettingstarted/whatisuml.htm.

28. Roux-Rouquie M, Caritey N, Gaubert L, et al. Using the unified modeling language (UML) to guide the systemic description of biological processes and systems. Biosystems. 2004;75(1-3):3-14

29. http://estime.spim.jussieu.fr/Menelas/.

30. Dhombres F, Vandenbussche PY, Rath A, et al. Orphanet projet OrphaONTO - première étape de l'ontologisation des bases de connaissances d'orphanet. Chambéry, France; 2011.

31. Noy NF, Mc Guinness DL. Ontology development 101:A guide to creating your first ontology. Stanford Knowledge Systems Laboratory Technical Report KSL-01-05 and stanford medical informatics technical report SMI-2001-0880; 2001

32. http://protege.stanford.edu/overview/protege-owl.html
33. Nishikawa S, Yamashita T, Imai T, et al. Thesaurus for histopathological findings in publically available reports of repeated-dose oral toxicity studies in rats for 156 chemicals. J Toxicol Sci. 2010;35(3):295-298.

34. Yang CC, Chih-Ping Wei CP, Li KW. Cross-lingual thesaurus for multilingual knowledge management. Decision Support Systems. 2008;45(3):596-605

35. Losee RM. Decisions in thesaurus construction and use. Information Processing \& Management. 2007;43(4):958-968.

36. http://www.nlm.nih.gov/mesh/MBrowser.html

37. http://www.cismef.org

38. http://www.thesaurusbuilder.com

39. Chinsembu KC. Model and experiences of initiating collaboration with traditional healers in validation of ethnomedicines for HIV/AIDS in Namibia. J Ethnobiol Ethnomed. 2009;5:30.

40. Simbo DJ. An ethnobotanical survey of medicinal plants in Babungo, Northwest Region, Cameroon. J Ethnobiol Ethnomed. 2010;6:8.

41. Togola A, Diallo D, Dembele S, et al. Ethnopharmacological survey of different uses of seven medicinal plants from Mali, (West Africa) in the regions Doila, Kolokani and Siby. J Ethnobiol Ethnomed. 2005;1:7.

42. Reyes-García V. The relevance of traditional knowledge systems for ethnopharmacological research:theoretical and methodological contributions. J Ethnobiol Ethnomed. 2010;6:32.

43. Jourdan C, Lefebvre C. Présentation. L'ethnolinguistique aujourd'hui. État des lieux. Anthropologie et Sociétés. 1999;23(3):pp. 5-13.

44. Zhou X, Chen S, Liu B, et al. Development of traditional Chinese medicine clinical data warehouse for medical knowledge discovery and decision support. Artif Intell Med. 2010;48(2-3):139-152.

45. Maiella S, Rath A, Angin C, et al. Orphanet and its consortium:where to find expert-validated information on rare diseases. Rev Neurol. 2013;169(Suppl 1):S3-S8.

46. Zhou X, Peng Y, Liu B. Text mining for traditional Chinese medical knowledge discovery:a survey. J Biomed Inform. 2010;43(4):650-660.

47. http://www.tei-c.org/release/doc/tei-p5-doc/en/html/index.html.

48. Sanou M. Investigations into the traditional practices in oral health in Burkina Faso with the Casablanca Faculty of Dental Medicine,2003. Morocco: HASSAN II Ain Chock University; 2003.

49. Jamal A, McKenzie K, Clark M. The impact of health information technology on the quality of medical and health care:a systematic review. HIM J. 2009;38(3):26-37.

50. Gunasekera V, Ernst E, Ezra DG. Systematic internet-based review of complementary and alternative medicine for glaucoma. Ophthalmology. 2008;115(3):435-439.e2

51. Boyer C, Baujard V, Geissbuhler A. Health on the net foundation, Switzerland. Stud Health Technol Inform. 2011;169:53-57.

52. http://www.churouen.fr/netscoring/

53. Xu W, Okada M. EBM metadata based on Dublin core better presenting validity of clinical trials. J Med Syst. 2007;31(5):337-343.

54. Cimino JJ. Desiderata for controlled medical vocabularies in the twentyfirst century. Methods Inf Med. 1998;37(4-5):394-403.

55. Manning CA, Vanrenen LJ. Bioenergetic Medicines East and West Acupuncture and Homeopathy, North Atlantic Books, California, USA; 1988. p. 111-247.

56. Knöss W, Chinou I. Regulation of medicinal plants for public healthEuropean community monographs on herbal substances. Planta Med. 2012;78(12):1311-1316 